\title{
Dynamic response of block copolymer wormlike micelles to shear flow
}

\author{
B Lonetti ${ }^{1}$, J Kohlbrecher ${ }^{2}$, L Willner ${ }^{1}$, J K G Dhont ${ }^{1}$ and \\ M P Lettinga ${ }^{1}$ \\ ${ }^{1}$ Institut für Festkörperforschung, Forschungszentrum Jülich, D-52425 Jülich, Germany \\ ${ }^{2}$ Laboratory for Neutron Scattering, ETH Zürich and Paul Scherrer Institut, 5232 Villigen PSI, \\ Switzerland
}

Received 2 May 2008

Published 10 September 2008

Online at stacks.iop.org/JPhysCM/20/404207

\begin{abstract}
The linear and nonlinear dynamic response to an oscillatory shear flow of giant wormlike micelles consisting of $\mathrm{Pb}-\mathrm{Peo}$ block copolymers is studied by means of Fourier transform rheology. Experiments are performed in the vicinity of the isotropic-nematic phase transition concentration, where the location of isotropic-nematic phase transition lines is determined independently. Strong shear-thinning behaviour is observed due to critical slowing down of orientational diffusion as a result of the vicinity of the isotropic-nematic spinodal. This severe shear-thinning behaviour is shown to result in gradient shear banding. Time-resolved small-angle neutron scattering experiments are used to obtain an insight into the microscopic phenomena that underlie the observed rheological response. An equation of motion for the order parameter tensor and an expression of the stress tensor in terms of the order parameter tensor are used to interpret the experimental data, both in the linear and nonlinear regimes. Scaling of the dynamic behaviour of the orientational order parameter and the stress is found when critical slowing down due to the vicinity of the isotropic-nematic spinodal is accounted for.
\end{abstract}

\section{Introduction}

Dispersions of surfactant wormlike micelles form a class of systems that has been intensively studied during the past two decades. Wormlike micellar systems sometimes exhibit extreme shear-thinning behaviour (Berret 2004), resulting in shear-induced structure formation like shear banding (Manneville 2008). Strong shear thinning is of practical interest, since often systems are required in practical applications that exhibit extreme differences in viscosity between the sheared and quiescent states. The reason for the popularity of wormlike micelles lies in their complex rheological behaviour like shear banding and chaotic response, which are connected to the thinning behaviour of these systems (Berret 2004). It is therefore important to understand the microscopic mechanism underlying the very strong shearthinning behaviour of wormlike micelles. There are several possible microscopic mechanisms that could be responsible for the occurrence of strong shear thinning (Cates and Candau 1990). One mechanism is related to the breaking and/or merging of worms. Scission due to shearing forces and merging of worms through stressed entanglement points (Briels et al 2004) can lead to strong shear thinning. Another possible mechanism for strong shear thinning is connected to the fact that wormlike systems can undergo an isotropicnematic (I-N) phase transition. Rotational diffusion close to I-N spinodal lines in the phase diagram is very slow, so that a relatively strong alignment on applying shear flow occurs. Such a strong increase in the degree of alignment leads in turn to strong shear thinning. We shall hereafter refer to the slowing down of rotational diffusion close to the I-N spinodals simply as 'critical slowing down'. By definition, the rotational diffusion coefficient at the spinodal changes sign, and is therefore zero at the spinodal, which implies very slow rotational Brownian motion.

For most studied surfactant wormlike micellar systems, the I-N transition occurs at relatively high volume fractions of around $10 \%$. At this high concentration the viscosity of the system is quite large, and moreover a transition to a gel phase can interfere. For CPLC/NaSal inbrine, for example, gelling occurs in the vicinity of the I-N transition on changing the temperature by just a few degrees. Furthermore, the I-N transition is only found under flow conditions. These features complicate detailed studies on the rheological response of wormlike micelles and its microscopic origin. We therefore study here a system that exhibits many of the properties 
of wormlike micellar systems that are responsible for their interesting rheological behaviour, but that does not have the above-mentioned complications of surfactant wormlike micellar systems. For an I-N transition to occur without flow, we need a system where the persistence length $l_{\mathrm{p}}$ is much larger than the thickness $d$ of the chains. The ratio $l_{\mathrm{p}} / d$ should be larger compared to typical values for wormlike micelles. A candidate system could be micelles formed from block copolymers. A well-studied system is the poly(butadiene)poly(ethylene oxide) ( $\mathrm{Pb}-\mathrm{Peo})$ diblock copolymer with a 5050 block composition in aqueous solution. The main advantage of this system is that it is very stiff, with a persistence length of around $500 \mathrm{~nm}$ and a diameter of $14 \mathrm{~nm}$. The contour length of the $\mathrm{Pb}-\mathrm{Peo}$ worms is around $1 \mu \mathrm{m}$. As a result of the large ratio $l_{\mathrm{p}} / d$ compared to common surfactant micellar systems, the diblock copolymer system shows an I$\mathrm{N}$ transition at a modest concentration of about $5 \%$, although the transition concentration has not been determined accurately yet (Won et al 1999). Other advantages of the $\mathrm{Pb}-\mathrm{Peo}$ system are that it is possible to tune the monomer-exchange kinetics between the polymers (Lund et al 2006) or its morphology (Denkova et al 2008) by using different solvent mixtures. Furthermore, these polymers are easily marked with fluorescent dyes, which enables their visualization with fluorescence microscopy. In a recent study Förster et al used this system, amongst others, for Rheo-SANS measurements, where stationary shear measurements were combined with small-angle neutron scattering (SANS) (Förster et al 2005). A feature of this diblock copolymer system that is probably not shared with micellar systems is that the polymers do not easily break and merge under flow. We thus focus on the microscopic mechanism mentioned above, related to critical slowing down of rotational diffusion close to the I-N transition.

In section 2, a well-known theoretical framework for the dynamics and rheological behaviour of stiff rods is summarized. This theory does not include flexibility of single polymer chains, but does include the slowing down of rotational diffusion due to the vicinity of the I-N spinodal. This theory will be used to assess the effect of the vicinity of the I-N transition on rheological response. A comparison of our experiments with predictions based on this theory can only be done on a qualitative level, since flexibility is neglected in the theory. After the materials section we describe a newly developed time-resolved SANS set-up, and the couette cells and rheometers that were used. In section 5 we first discuss the flow curve of the system and determine the corresponding flow profiles. It is also shown in this section how the (nonequilibrium) binodal line can be found from shear step-down experiments. Then we discuss SANS experiments on quiescent and stationary sheared systems, which we need as an input in the last subsection on dynamic experiments. In the latter subsection we connect the time-resolved SANS measurements with Fourier transform rheology results. The spinodal point is determined in order to establish whether the concept of critical slowing down indeed applies.

\section{Theory}

\subsection{Concentration dependence of the rotational diffusion coefficient}

On approach of the isotropic-nematic (I-N) spinodal, the collective rotational diffusion coefficient vanishes and becomes negative in the unstable part of the phase diagram. As will be discussed later, this rotational diffusion coefficient describes the dynamics of small perturbations of the orientational order parameter from its value in a stationary state. For a system of very long and thin, rigid rods with repulsive interactions that have a range that is small compared to the length of the rods, critical slowing is described by the equation of motion for the orientational order parameter tensor $\mathbf{S} \equiv\langle\hat{\mathbf{u}} \hat{\mathbf{u}}\rangle$, where $\hat{\mathbf{u}}$ is the unit vector along the long axis of a rod, which specifies the orientation of the rod, and where the brackets indicate ensemble averaging. Starting from the Smoluchowski equation for rod-like colloids with hard-core interactions, an equation of motion for $\mathbf{S}$ can be derived (Dhont and Briels 2003a), which is similar to the Doi-Edwards equation of motion (Doi and Edwards 1986):

$$
\begin{aligned}
\frac{\mathrm{d}}{\mathrm{d} t} \mathbf{S} & =-6 D_{\mathrm{r}}\left\{\mathbf{S}-\frac{1}{3} \hat{\mathbf{I}}+\frac{L}{D} \varphi\left(\mathbf{S}^{(4)}: \mathbf{S}-\mathbf{S} \cdot \mathbf{S}\right)\right\} \\
& +\dot{\gamma}\left\{\hat{\boldsymbol{\Gamma}} \cdot \mathbf{S}+\mathbf{S} \cdot \hat{\boldsymbol{\Gamma}}^{\mathrm{T}}-2 \mathbf{S}^{(4)}: \hat{\mathbf{E}}\right\}
\end{aligned}
$$

where $D_{\mathrm{r}}$ is the rotational diffusion coefficient at infinite dilution, $L$ is the length of the rods, $d$ their core diameter, $\varphi$ is the volume fraction of rods, $\dot{\gamma}$ is the shear rate, $\hat{\Gamma}$ is the velocity-gradient tensor and $\hat{\mathbf{E}}=\frac{1}{2}\left[\hat{\boldsymbol{\Gamma}}+\hat{\boldsymbol{\Gamma}}^{\mathrm{T}}\right]$ is the symmetrized velocity-gradient tensor (where the superscript ' $\mathrm{T}$ ' stands for 'transpose'). Furthermore, $\mathbf{S}^{(4)} \equiv\langle\hat{\mathbf{u}} \mathbf{u} \hat{u} \hat{\mathbf{u}}\rangle$ is a fourth-order tensor. A closure relation that expresses contractions of the form $\mathbf{S}^{(4)}$ : $\mathbf{M}$ in terms of $\mathbf{S}$ for arbitrary second rank tensors $\mathbf{M}$ is discussed in (Dhont and Briels 2003a):

$$
\begin{gathered}
\langle\hat{\mathbf{u}} \hat{\mathbf{u}} \hat{\mathbf{u}} \hat{\mathbf{u}}\rangle: \mathbf{M}=\frac{1}{5}\{\mathbf{S} \cdot \overline{\mathbf{M}}+\overline{\mathbf{M}} \cdot \mathbf{S}-\mathbf{S} \cdot \mathbf{S} \cdot \overline{\mathbf{M}} \\
-\overline{\mathbf{M}} \cdot \mathbf{S} \cdot \mathbf{S}+2 \mathbf{S} \cdot \overline{\mathbf{M}} \cdot \mathbf{S}+3 \mathbf{S S}: \overline{\mathbf{M}}\},
\end{gathered}
$$

where $\overline{\mathbf{M}}=\frac{1}{2}\left[\mathbf{M}+\mathbf{M}^{\mathrm{T}}\right]$ is the symmetric part of the tensor M. For simple shear flow, the velocity-gradient tensor has the form

$$
\hat{\boldsymbol{\Gamma}}=\left(\begin{array}{lll}
0 & 1 & 0 \\
0 & 0 & 0 \\
0 & 0 & 0
\end{array}\right),
$$

which corresponds to a flow in the $x$ direction with its gradient in the $y$ direction.

The largest eigenvalue $\lambda$ of $\mathbf{S}$, the 'orientational order parameter', is a measure of the degree of alignment (for the isotropic state $\lambda=1 / 3$ and for a perfectly aligned state, $\lambda=$ 1). In order to illustrate critical slowing down of orientational diffusion, we consider first an isotropic state which is slightly perturbed. The equation of motion for a small perturbation $\delta \lambda$ of $\lambda=1 / 3$ in the isotropic state, in the absence of flow, is readily obtained from equation (1) together with the closure relation (2):

$$
\frac{\mathrm{d} \delta \lambda}{\mathrm{d} t}=-6 D_{\mathrm{r}}\left\{1-\frac{1}{4} \frac{L}{d} \phi\right\} \delta \lambda=-6 D_{\mathrm{r}}^{\mathrm{eff}} \delta \lambda
$$


where

$$
D_{\mathrm{r}}^{\mathrm{eff}}=D_{\mathrm{r}}\left\{1-\frac{1}{4} \frac{L}{d} \phi\right\}
$$

is the effective rotational diffusion coefficient. Hence,

$$
\delta \lambda(t)=\delta \lambda(t=0) \exp \left\{-6 D_{\mathrm{r}}^{\mathrm{eff}} t\right\}
$$

From equation (5) it can be seen that $D_{\mathrm{r}}^{\text {eff }} \rightarrow 0$ as $(L / d) \varphi \rightarrow$ 4. Collective rotational diffusion thus becomes very slow on approach of the spinodal concentration where $(L / d) \varphi=4$. For larger concentrations, where $D_{\mathrm{r}}^{\text {eff }}<0$, the isotropic state is unstable, and the initially small orientational order parameter increases in time. In the presence of shear flow, the above analysis must be done numerically, since the unperturbed (stable or unstable) stationary state under shear flow is not known analytically. The effective rotational diffusion coefficient is now a tensorial quantity rather than a scalar as for the isotropic state discussed above. The phenomenon of critical slowing down, however, is unchanged: rotational diffusion becomes very slow on approach of the spinodal (where at least one of the eigenvalues of the rotational diffusion tensor changes sign). This slowing down of rotational diffusion has pronounced effects on the shear-thinning behaviour, as will be discussed later.

\subsection{Dynamic response of stress and orientational order}

From microscopic considerations, an expression for the stress tensor $\Sigma$ can be obtained (Dhont and Briels 2003b), which is similar to an earlier derived expression by Doi and Edwards (Doi and Edwards 1986):

$$
\begin{aligned}
\boldsymbol{\Sigma}_{D} & =2 \eta_{0} \dot{\gamma}\left[\hat{\mathbf{E}}+\frac{(L / D)^{2}}{3 \ln \{L / D\}} \varphi\right. \\
& \left.\times\left\{\hat{\boldsymbol{\Gamma}} \cdot \mathbf{S}+\mathbf{S} \cdot \hat{\boldsymbol{\Gamma}}^{\mathrm{T}}-\mathbf{S}^{(4)}: \hat{\mathbf{E}}-\frac{1}{3} \hat{\mathbf{I} S}: \hat{\mathbf{E}}-\frac{1}{\dot{\gamma}} \frac{\mathrm{d} \mathbf{S}}{\mathrm{d} t}\right\}\right] .
\end{aligned}
$$

For an oscillatory shear flow, the shear rate $\dot{\gamma}$ in equations (1) and (7) is time-dependent:

$$
\dot{\gamma}(t)=\dot{\gamma}_{0} \cos \{\omega t\}
$$

where $\dot{\gamma}_{0}=A \omega$ is the shear amplitude, with $A$ the strain amplitude and $\omega$ the frequency of oscillation.

The linear and nonlinear response of suspensions or rigid rods, within the approximations involved in the theory, can be obtained from numerical solutions of equations (1) and (7) (Dhont and Briels 2003a). In particular, dynamic response functions can be obtained from a Fourier analysis of the time dependence of the stress tensor after transients have relaxed. For sufficiently large shear rates, higher-order nonlinear response functions come into play. For these higher shear amplitudes, the time-dependent stress tensor must be Fourier-expanded as

$$
\boldsymbol{\Sigma}_{D}=2 \dot{\gamma}_{0} \hat{\mathbf{E}} \sum_{n=0}^{\infty}|\eta|_{n} \sin \left(n \omega t+\delta_{n}\right),
$$

where $|\eta|_{n}$ and $\delta_{n}$ are the amplitude and phase shift of the Fourier components, respectively. Similarly, the scalar orientational order parameter will respond in a nonlinear fashion, so that

$$
P_{2}(t)=\sum_{n}^{\infty}\left|P_{2}\right|_{n} \cos \left(\omega t+\epsilon_{n}\right),
$$

where $P_{2}=\frac{1}{2}[3 \lambda-1]$ (as before, $\lambda$ is the largest eigenvalue of $\mathbf{S}$ ). It should be mentioned that in scattering experiments only projections of the orientational order parameter tensor are probed. In that case, $P_{2}$ in equation (10) does not correspond to the largest eigenvalue of $\mathbf{S}$, but only to the corresponding projection of $\mathbf{S}$. In the experiments described in this paper the vorticity-flow plane is probed, for which it is readily shown from equation (1) by expanding $\mathbf{S}$ for small shear rates that the leading term in shear rate varies like $\sim \dot{\gamma}^{2}$. The time dependence of the experimentally determined orientational order parameter term has therefore the double frequency of the applied shear flow.

One may ask about the shear rate beyond which nonlinear response is expected, and beyond which a frequency phase shift will be found. An analysis of the equation of motion (1) and the expression (7) for the stress tensor for the isotropic state and to leading order in nonlinearity reveals that the socalled effective Peclet number:

$$
P e_{\mathrm{eff}}=\dot{\gamma}_{0} / D_{\mathrm{r}}^{\mathrm{eff}},
$$

and the effective Deborah number:

$$
\Omega_{\mathrm{eff}}=\omega / D_{\mathrm{r}}^{\mathrm{eff}}
$$

measure the nonlinearity and phase shift. Here, the effective rotational diffusion coefficient is given in equation (5).

\section{Material}

In this study we used a symmetric $\mathrm{Pb}-\mathrm{Peo}$ block copolymer prepared by living anionic polymerization; the synthesis follows a two-step procedure since the polymerization conditions for ethylene oxide are different from those for butadiene. Details of the two-step procedure can be found in an earlier publication (Allgaier et al 1997). The Pb-Peo block copolymer was characterized by size exclusion chromatography (SEC) using a mixture of tetrahydrofuran/dimethylacetamide $90 / 10 \mathrm{v} / \mathrm{v}$ as eluant. The polydispersity, Mw/Mn, of the block copolymer was smaller than 1.04. No signs of PEO and PB homopolymers were found in the SEC chromatograms. Absolute molecular weights were determined by $1 \mathrm{H}-\mathrm{NMR}$ measurements in $\mathrm{CDCl}_{3}$. Thereby, the signal of the t-butyl initiator group was taken as an internal reference. The number average molecular weights, $\mathrm{Mn}$, are $2.6 \mathrm{~kg} \mathrm{~mol}^{-1}$ for PB and $2.64 \mathrm{~kg} \mathrm{~mol}^{-1}$ for PEO. Polymer solutions were prepared by dissolving the polymer in $\mathrm{D}_{2} \mathrm{O}$ (Chemotrade, $\% D=99.8 \%$ ) and, in order to guarantee its complete dissolution, especially in the case of high concentration samples, they were kept for half an hour at $56^{\circ} \mathrm{C}$ and then left to cool down slowly to the ambient temperature. When not specified otherwise, the concentrations will be expressed as a weight fraction. 

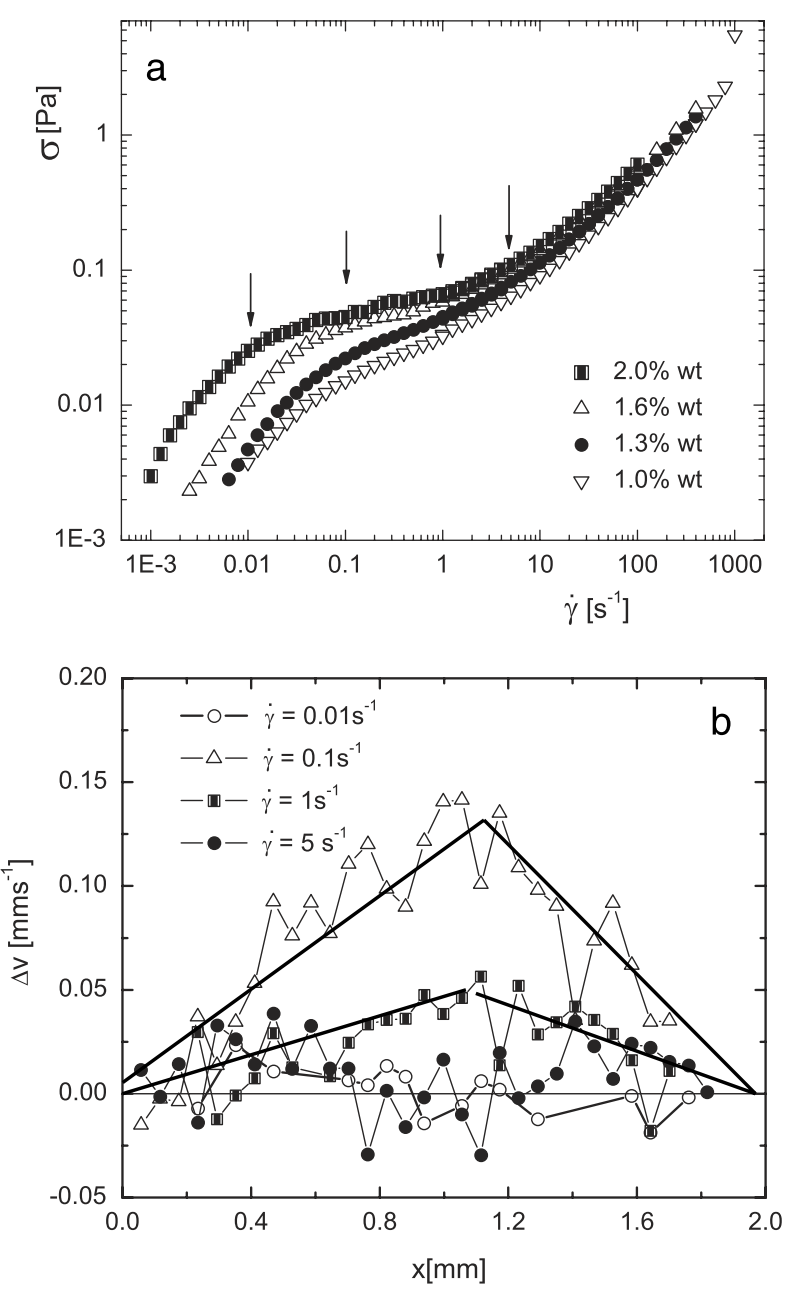

Figure 1. (a) Flow curves for different $\mathrm{Pb}-\mathrm{Peo}$ concentrations. (b) The relative flow velocity $\Delta v(x)=V(x)-\dot{\gamma} x$ throughout the gap of the couette cell for various applied shear rates as indicated by the arrows in (a): $0.01,0.1,1$ and $5 \mathrm{~s}^{-1}$. The lines indicate two shear bands.

\section{Experimental details}

SANS experiments have been performed at the SANS I instrument at the SINQ spallation source at the Paul Scherrer Institute (PSI) in Villigen, Switzerland (Kohlbrecher and Wagner 2000). We used thermal neutrons of wavelength $\lambda=0.8 \mathrm{~nm}$ with a wavelength spread $\Delta \lambda / \lambda$ of about 0.1 . The data analysis was performed using the BerSANS software package (Keiderling 2002). A standard water sample was used for calibration of absolute scattering intensities and also to account for non-uniform detector efficiency. For the RheoSANS experiments a Rheowis strain-controlled rheometer with a couette type shear cell (bob: $48 \mathrm{~mm}$ radius, cup: $50 \mathrm{~mm}$ radius) was placed in the neutron beam in the so-called radial configuration. In this configuration the neutron beam passes through the centre of the sapphire cell, transparent for neutrons, and is parallel to the gradient direction so that the flowvorticity plane is probed by the $2 \mathrm{D}$ detector. The accessible torque range is between $10^{-7}$ and $0.046 \mathrm{~N} \mathrm{~m}$, the frequency range between $5 \times 10^{-3}$ and $10 \mathrm{~Hz}$ and the amplitude range between $5 \times 10^{-2}$ and 45 . Both steady state and oscillatory experiments were performed. In order to probe the timedependent structural changes with SANS under oscillating shear, a stroboscopic data acquisition scheme, implemented on the SANS-1 instrument, has been used. The electronics of the rheometer supplies a low and high signal depending on the turning direction. The falling edge of this rectangular signal has been used to trigger the data acquisition of the scattered neutrons, producing histograms of $128 \times 128$ pixels of $0.75 \times 0.75 \mathrm{~cm}^{2}$ spatial resolution and at least $n=100$ time channels of widths $\Delta t=(n \times \omega / 2 \pi)^{-1}$, where $\omega / 2 \pi$ is the frequency of the applied oscillating shear. The time of flight $t_{\text {tof }}$ of the scattered neutrons between sample and detector has been corrected to obtain the exact phase between applied shear and scattered neutrons. However, this correction can be practically neglected as the applied shear frequencies are much lower than $1 / t_{\text {tof }}$. Before starting the neutron data acquisition the rheometer was oscillating for several cycles to ensure that no transient effects were measured. To obtain sufficient counting statistics for each time channel, the histograms of many shear cycles were summed up over a time going from $1 \mathrm{~h}$ to $15 \mathrm{~min}$ for the lowest and highest concentration, respectively. With this technique the temporal evolution of the structural alignment of the diblock copolymers during a whole shear cycle could be measured.

Fast Fourier transform rheological experiments were performed on a strain-controlled rheometer (ARES, TA instruments), using a couette geometry (bob: $32 \mathrm{~mm}$ radius, cup: $34 \mathrm{~mm}$ radius). The stress response to dynamic strain experiments has been simultaneously recorded with a Analog Digital Card and analysed with fast Fourier transform software as described in (Wilhelm et al 1998). The same instrument was used for step-rate experiments and to obtain flow curves. Spatially resolved velocity profiles were measured on a homebuilt heterodyne dynamic light scattering set-up using a closed, transparent couette cell (2 mm gap), see e.g. (Salmon et al 2003).

\section{Results and discussion}

\subsection{Flow curve and step-down rheology}

The $\mathrm{Pb}-\mathrm{Peo}$ block copolymer under study forms wormlike micelles in water solution. As molecular wormlike micelles, consisting of surfactant molecules, these giant wormlike micelles show a pronounced shear-thinning behaviour. Figure 1(a) shows the stress as a function of the shear rate for $\mathrm{Pb}-\mathrm{Peo}$ solutions with volume fraction between $1 \%$ and $2 \%$. These concentrations lie close to the suggested literature value for the I-N transition (Won et al 1999), but are still in the isotropic phase. All the curves in figure 1(a) exhibit a shear-thinning region which extends to lower shear rates with increasing volume fraction of micelles, while the corresponding stress plateau becomes flatter. For the sample with the highest concentration, i.e. $[\mathrm{Pb}-\mathrm{Peo}]=2 \%$, we tested if the sample shows shear banding, as is expected for extreme shear-thinning samples (Dhont and Briels 2008, Olmsted 2008). A few typical velocity profiles relative to the applied shear rate within the gap of the couette cell are 

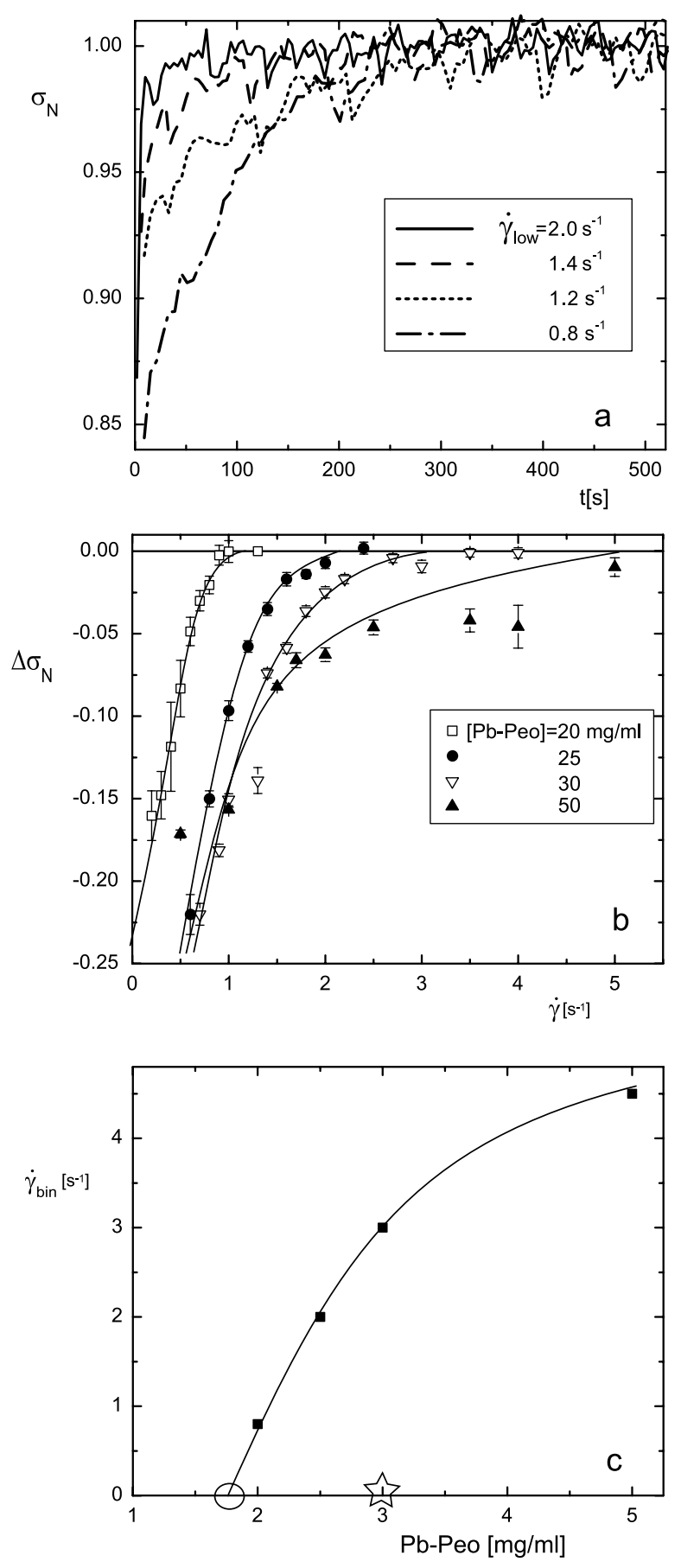

Figure 2. (a) The response of the normalized stress $\sigma_{\mathrm{N}}(t)=\sigma(t) / \sigma(t \rightarrow \infty)$ to shear rate quenches from the fully nematic state into the biphasic region. The initial shear rate was $\dot{\gamma}=7 \mathrm{~s}^{-1}$ and the low shear rates were varied between $\dot{\gamma}=0.8 \mathrm{~s}^{-1}$ (bottom) and $\dot{\gamma}=2.0 \mathrm{~s}^{-1}$ (top). (b) The magnitude of the stress response $\Delta \sigma_{\mathrm{N}}$, obtained from the fit to $\sigma_{\mathrm{N}}(t)=1-\Delta \sigma_{\mathrm{N}} \mathrm{e}^{-t / \tau}$ as a function of the shear rate. Lines are guides to the eye. (c) The resulting binodal points obtained from the shear rate in (b) where $\Delta \sigma_{\mathrm{N}}$ becomes zero. The circle indicates the equilibrium I-N binodal, that is, the binodal point in the absence of flow. The line is a guide to the eye, representing the non-equilibrium binodal. The open star indicates the location of the spinodal at zero shear rate.

plotted in figure 1(b), as obtained from spatially resolved heterodyne light scattering measurements. Shear banding is observed between 0.1 and $0.75 \mathrm{~s}^{-1}$, which corresponds to the flat region in the flow curve in figure 1(a). At the lowest investigated shear rate, $0.01 \mathrm{~s}^{-1}$, the velocity profile is linear (see figure 1(b)). Increasing the shear rate to $0.1 \mathrm{~s}^{-1}$, inside the stress plateau region, a banded structure can be recognized and the velocity profile shows a characteristic kink, as can be seen from figure 1(b). In the investigated overall shear-rate range, the average shear rate in the high shear-rate band is twice that of the lower shear-rate band. The fraction of the gap occupied by the high shear-rate band increases with the overall shear rate, and for shear rates higher than $1 \mathrm{~s}^{-1}$ the low shear-rate band disappears and a linear profile is re-established.

In order to locate the isotropic-nematic binodal, i.e. the point where the isotropic phase becomes metastable, rheology is a very useful tool as the viscosity of the micellar solution is very sensitive to the local orientation of the worms. To exploit the large difference between the viscosity of the isotropic and nematic phases, we performed step-down experiments in the concentration region between $2 \%$ and $5 \%$. As we have shown in an earlier paper on rod-like viruses (Lettinga and Dhont 2004), the viscosity of the system will increase in time when the system is quenched from a high shear rate, where the nematic phase is stable, to a lower shear rate, where the nematic phase becomes meta- or unstable. Figure 2(a) shows an example of the normalized stress $\sigma_{\mathrm{N}}(t)=\sigma(t) / \sigma(t \rightarrow \infty)$ (where $\sigma$ is the shear stress) as a function of time after the shear rate was quenched from $7 \mathrm{~s}^{-1}$ to a final value ranging from 2 to $0.8 \mathrm{~s}^{-1}$. The curves are fitted to a single exponential $\sigma_{\mathrm{N}}(t)=1-\Delta \sigma_{\mathrm{N}} \mathrm{e}^{-t / \tau}$, where $\Delta \sigma_{\mathrm{N}}$ depends on the fraction of the formed isotropic phase, which tends to zero at the binodal point. Thus, for each concentration the binodal point was determined as the shear rate at which $\Delta \sigma_{\mathrm{N}}$ vanishes (see figure 2(b)). The resulting binodal points are plotted in figure 2(c). This figure constitutes the low concentration branch of the non-equilibrium binodal for the $\mathrm{Pb}-\mathrm{Peo}$ block copolymer system. The equilibrium I-N binodal, in the absence of flow, is found to be located at $[\mathrm{Pb}-\mathrm{Peo}]=$ $1.7 \pm 0.1 \%$. The open star in figure 2 indicates the location of the spinodal at zero shear rate. How this spinodal point was determined will be discussed later.

As we are dealing with a system that can also be described as flexible rods, we know from Chen (1993), for example, that the $\mathrm{I}-\mathrm{N}$ phase coexistence region is very broad and thus the location of the I-N spinodal can be found at a significantly higher concentration than the $\mathrm{I}-\mathrm{N}$ binodal. In the case of rigid rods, the collective rotational diffusion becomes very small on approach of the spinodal point, as discussed in section 2. For semi-flexible chains, the rotational motion of the Kuhn segments will become very slow on the approach of the spinodal. We will now employ dynamic experiments to access this slowing down. We want to do this not only on the macroscopic level, i.e. by rheology, but also on the microscopic level, in order to establish a link between the behaviour of Kuhn segments and the measured stress in the system.

\subsection{SANS on quiescent and stationary sheared samples}

The quiescent dispersion of $\mathrm{Pb}-\mathrm{Peo}$ micelles has an angleaveraged scattering pattern as plotted in figure 3(a). At low 

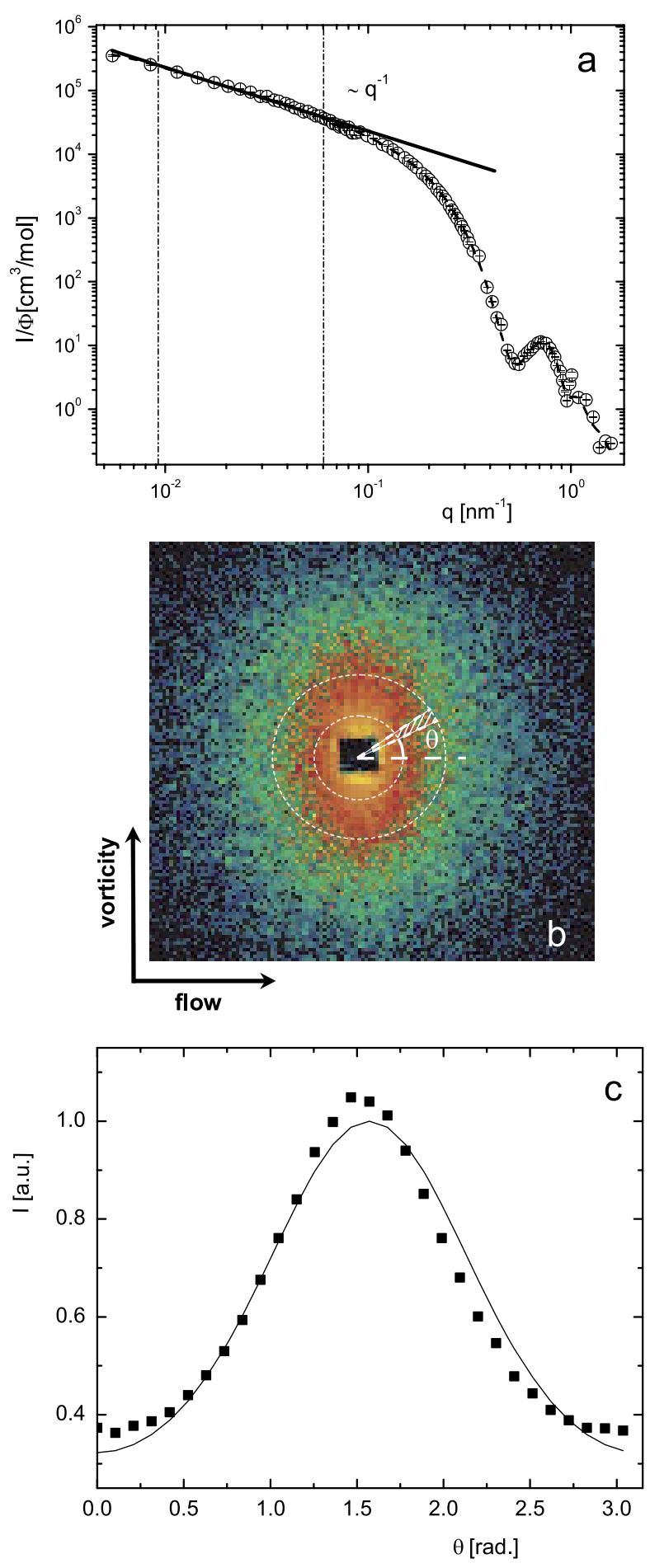

Figure 3. (a) Angle-averaged SANS curve at zero shear. The full line indicates the $q$ range where a $q^{-1}$ dependence is found, typical for rods. (b) Scattering pattern of $1 \% \mathrm{~Pb}-\mathrm{Peo}$ in deuterated water at shear rate $\dot{\gamma}=1 \mathrm{~s}^{-1}$ in the flow-velocity plane. The dashed lines indicate the $Q$ range that is used to obtain the azimuthal intensity profile as plotted in (c). Here $\theta$ is the angle with the shear flow and the full line indicates a fit to equation (13).

(This figure is in colour only in the electronic version)

$Q$ values, the scattering curve shows a $I \sim q^{-1}$ dependence, typical for rods. The transition from $I \sim q^{-1}$ to a $I \sim q^{-2}$ dependence that is expected for wormlike micelles is outside
Table 1. Structural micelle characteristics as obtained from fitting of the SANS curve in figure 3.

\begin{tabular}{llll}
\hline$N_{\text {ag }}\left(\mathrm{nm}^{-1}\right)$ & $\sigma_{\text {core }}(\mathrm{nm})$ & $\sigma_{\text {shell }}(\mathrm{nm})$ & $\beta$ \\
\hline 26.5 & 6.4 & 7.8 & 3.65 \\
\hline
\end{tabular}

the experimental window. This shows that the persistence length of the worms is at least $500 \mathrm{~nm}$, in agreement with previous experiments on the same system (Won et al 1999). For this reason the data could be fitted with the form factor of a long cylinder. The details of the fitting procedure are beyond the scope of this paper and will be described elsewhere. The main point is that the cylinders are assumed to have a uniform core and a shell with an exponential density profile, i.e. density $\sim(1-r) \mathrm{e}^{(-\alpha r)}$, where $r=\frac{r-\sigma_{\text {core }}}{\sigma_{\text {shell }}}$. From the fitting, the core and shell radii $\sigma_{\text {core }}$ and $\sigma_{\text {shell }}$, the aggregation number per unit length and the exponent $\alpha$ have been obtained. The numerical values of these parameters are given in table 1 . The values for the cylinder cross section is in agreement with that already reported in the literature (Won et al 1999).

Figure 3(b) shows a typical scattering pattern of $\mathrm{Pb}-\mathrm{Peo}$ under shear conditions (with $\dot{\gamma}=1 \mathrm{~s}^{-1}$ ), which shows the shear-induced anisotropic structure. This can be more clearly seen in the azimuthal intensity profile, as plotted in figure 3(c), which is obtained from the part of the scattering pattern in figure 3(b) where the scattered intensity is proportional to $q^{-1}$ (the area in between the circles in figure 3(b)). Assuming a Maier-Saupe type of orientation distribution function, the azimuthal scattered intensity $I(Q, \theta)$ from the nematic phase is generally well described by (Picken et al 1990)

$$
I(Q, \theta) \sim \exp \left\{\beta P_{2}(\theta)-1\right\},
$$

where the parameter $\beta$ describes the width of the intensity profile and $P_{2}$ is the second-order Legendre polynomial. The solid line in figure 3(c) shows an example of a fit of the expression in equation (13) with the experimental data as obtained from the scattering pattern in figure $3(\mathrm{~b})$. The scalar order parameter $\left\langle P_{2}(\theta)\right\rangle$ can then be calculated from

$$
\left\langle P_{2}(\theta)\right\rangle=\frac{\int_{0}^{\pi} \exp \left\{\beta P_{2}(\theta)\right\} P_{2}(\theta) \sin (\theta) \mathrm{d} \theta}{\int_{0}^{\pi} \exp \left\{\beta P_{2}(\theta)\right\} \sin (\theta) \mathrm{d} \theta} .
$$

In this way, the order parameter $\left\langle P_{2}(\theta)\right\rangle$ can be obtained from scattering data for each shear rate at different concentrations. As expected, flow-induced orientation of the cylindrical micelles is observed.

In Förster et al (2005) it is suggested that the shear viscosity is a universal function when plotted against the orientational order parameter, independent of concentration. We indeed find such a behaviour for our $\mathrm{Pb}-\mathrm{Peo}$ system, as can be seen from figure 4. For the two concentrations of 1 and $2 \%$, the two curves fall on top of each other. Contrary to Förster et al (2005), we do not find a linear dependence of the viscosity on the order parameter, probably due to the fact that we also used data at shear rates lower than those where the stress plateau occurs. 


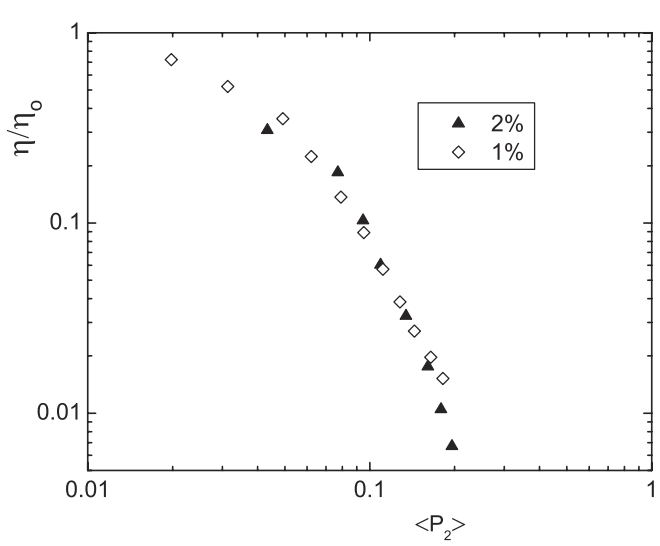

Figure 4. The scaled viscosity $\eta$ (with $\eta_{0}$ the viscosity at zero shear rate) versus the orientational order parameter $\left\langle P_{2}\right\rangle$.

\subsection{Dynamic experiments}

Oscillatory shear-rate experiments were performed for concentrations lower and around the I-N equilibrium binodal point, i.e. between 0.5 and $2 \%$. As for steady-state measurements, the order parameter $\left\langle P_{2}\right\rangle$ can be calculated from SANS experiments according to equations (13) and (14) at each point in time during an oscillation. In this way we probe the time dependence of the orientational order parameter $\left\langle P_{2}\right\rangle$. In order to compare and relate the orientational response with the change in the stress the stress response was also recorded and analysed by fast Fourier transform rheology experiments on samples in a somewhat broader concentration range between 0.5 and $2.5 \%$.

In figure 5 we plot the time-dependent response of $\left\langle P_{2}\right\rangle$ ((a) and (b)) and the stress ((c) and (d)) of a $2 \%$ sample. In figures 5(a) and (c), the response for different shear amplitudes $\dot{\gamma}_{0}$ (see equation (8)) is shown, where the maximum shear rate during an oscillation is kept constant by adjusting the frequency. In figures 5(b) and (d), the response for different frequencies is shown, where again the maximum shear rate during an oscillation is kept constant, but now by adjusting the shear amplitude. The first thing to note is that the order parameter oscillates with twice the frequency of the applied shear rate, even for low shear rates where the stress response is linear in the shear rate. The reason for this is that the scattering experiments probe the flow-vorticity plane, so that the measured order parameter characterizes the orientational order within that plane. As already discussed in section 2, there is no linear response of the order parameter in this plane and the leading response is quadratic in the shear rate. This results in the double-frequency response of the probed projection of orientational order. The experimental trends are in good qualitative agreement with the theoretical calculations based on equations (7) and (1), as can be seen from figure 6, where figure 6(a) should be compared to the experimental results in figure 5(b), and figures 6(b)-5(d). The theoretical curves have the same form as the experimental curves, exhibiting similar trends on changing frequency and shear amplitude. In order to quantify the dynamic response we analyse this response on the basis of the Fourier modes as given in equations (9) and (10) for the stress and (flow-vorticity projected) orientational order parameter $\left\langle P_{2}\right\rangle$, respectively. The experimental phase shifts for the $2 \%$ sample are shown in figures $7(a),(c)$ and (e). The Fourier amplitude ratios that measure the departure from linear response, $\left|P_{2}\right|_{4} /\left|P_{2}\right|_{2}$ for $\left\langle P_{2}\right\rangle$ and $|\eta|_{3} /|\eta|_{1}$ for the stress, are plotted in figures 7(b), (d) and (f).

As mentioned in the theory section 2 , the rate at which a dispersion of rods relaxes close to the spinodal point is determined by the effective diffusion coefficient $D_{\text {eff }}$ given by equation (5). There are two unknown parameters in this equation, namely the spinodal concentration, i.e. the concentration where $\frac{L}{d} \phi=4$, and the rotational diffusion at infinite dilution $D_{\mathrm{r}}$. When critical slowing down is at the origin of the difference in dynamic response for various concentrations, we should find scaling when response functions are plotted against effective quantities, like the effective Peclet number in equation (11) and the effective Deborah number in equation (12). In order to test such a scaling for relatively low shear rates, we need to know the concentration where the I-N spinodal in the absence of flow is located. In view of our expression (5) for the effective rotational diffusion coefficient, we will use the following similar form for the effective diffusion coefficient of the $\mathrm{Pb}-$ Peo system:

$$
D_{\mathrm{r}}^{\mathrm{eff}}=D_{\mathrm{r}}\{1-[\mathrm{Pb}-\mathrm{Peo}] / C\},
$$

where, as before, $[\mathrm{Pb}-\mathrm{Peo}]$ is the concentration of $\mathrm{Pb}-\mathrm{Peo}$ and $C$ is a scaling parameter that determines the location of the I-N spinodal. For a given value of the parameter $C$, the effective Peclet and Deborah numbers are calculated from equations (11) and (12).

As can be seen from figure 9, all experimental data for phase shifts and nonlinear response functions collapse on a single curve when $C$ is taken equal to 3 . This is true for both the stress response as well as for the response of the orientational order parameter $\left\langle P_{2}\right\rangle$ (projected on the flowvorticity plane). This is in accord with the idea that the concentration dependence of the response of both orientational order as well as the stress is related to critical slowing down. Thus, in terms of polymer concentration, the spinodal point is located at $[\mathrm{Pb}-\mathrm{Peo}]_{\text {spin }}=3 \%$. The spinodal point is indicated by the open star in figure 2(c). This spinodal concentration seems to be in accord with the somewhat lower binodal concentration of $1.7 \%$ in the absence of flow.

The spinodal concentration can be estimated from the length, thickness and volume fraction of the wormlike micelles, neglecting the effect of the flexibility. As discussed before, the structural parameters of the wormlike micelles have been derived from fitting of scattering data (see table 1). In this way we can estimate the location of the I-N spinodal by equating both expressions (5) and (15) for the effective diffusion coefficient. Since $\phi=2.73 \times[\mathrm{Pb}-\mathrm{Peo}], d=$ $14.2156 \mathrm{~nm}$ and $L=1 \mu \mathrm{m}$ ( $L$ obtained from DLS and microscopy data, to be published) we find that

$$
[\mathrm{Pb}-\mathrm{Peo}]_{\text {spin }}=\frac{4}{2.73} \times \frac{d}{L}=2.1 \%,
$$



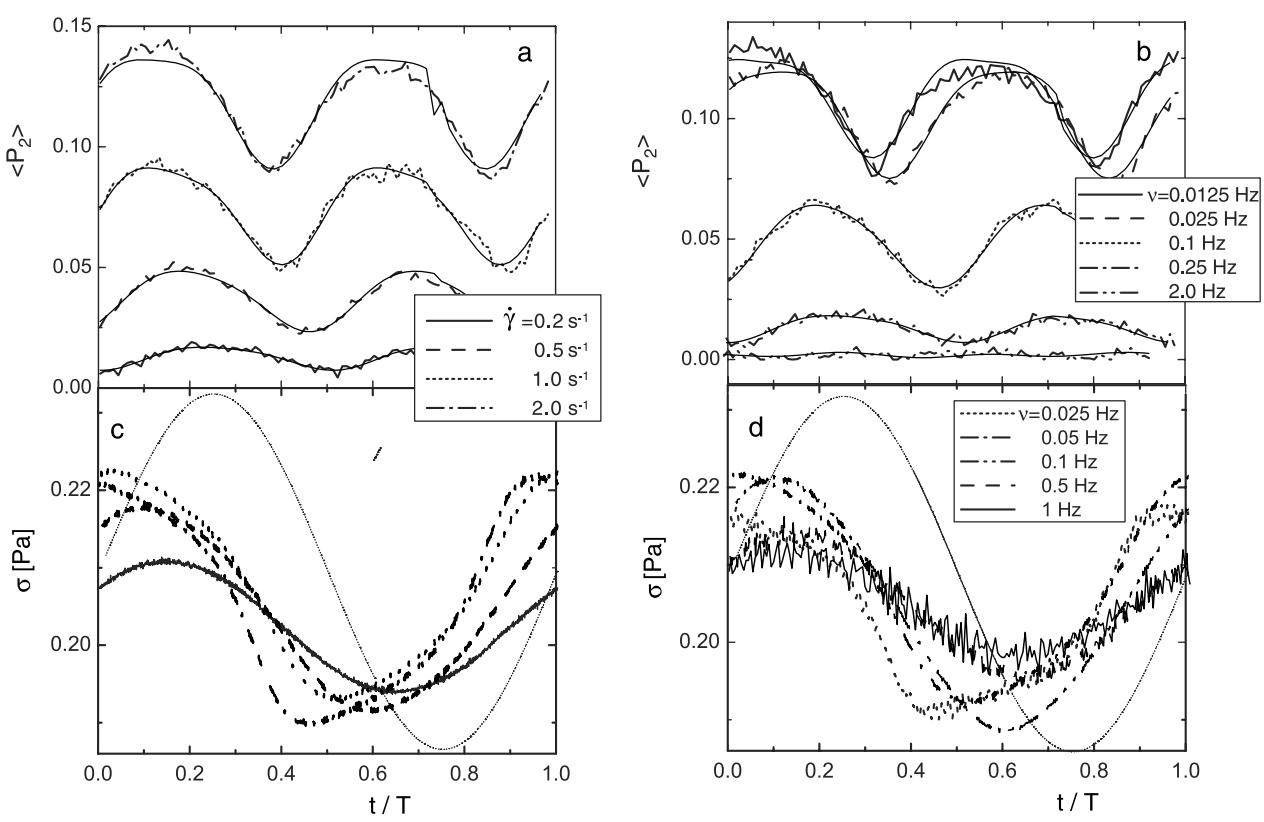

Figure 5. Time-dependent response of the orientational order parameter $\left\langle P_{2}\right\rangle((\mathrm{a})$ and (b)) and the stress ((c) and (d)) to an oscillatory shear flow at a shear rate of $\dot{\gamma}_{\max }=1.0 \mathrm{~s}^{-1}$ ((a) and (c)) and a frequency of $0.05 \mathrm{~Hz}((\mathrm{~b})$ and (d)) at a concentration of $2 \% \mathrm{~Pb}-\mathrm{Peo}$. The thin dotted curves indicate the applied shear rate. The time $t$ is scaled with the period $T$ of oscillation.
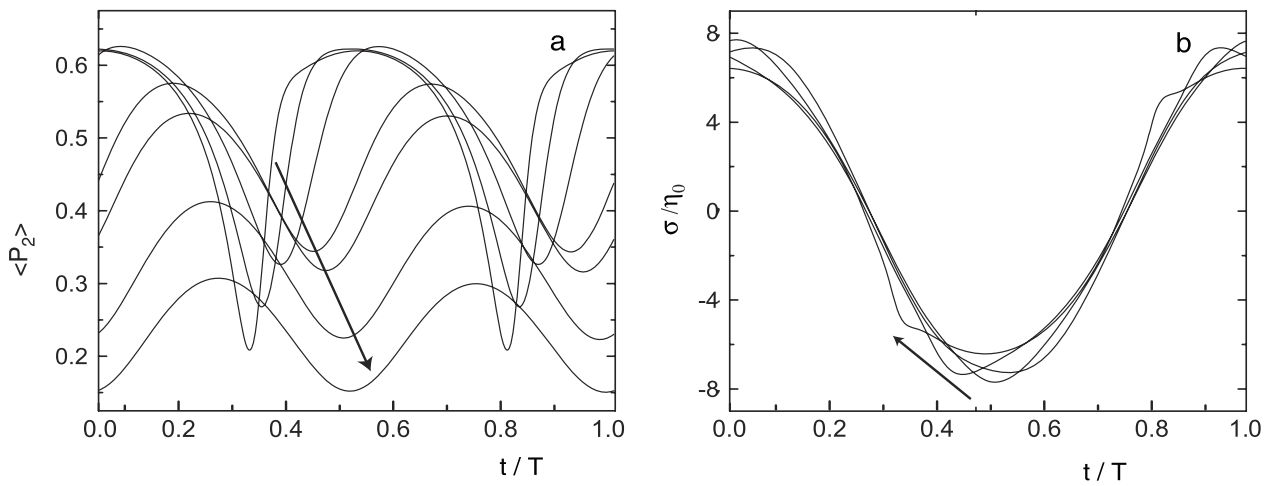

Figure 6. Theoretical predictions for the response of (a) the stress and (b) the order parameter $\left\langle P_{2}\right\rangle$ for $\Omega$ varying from 3 to 60 . The arrows indicates increasing $\Omega$. The effective Peclet number is $\mathrm{Pe}_{\mathrm{eff}}=75$ and the concentration is $\frac{L}{d} \varphi=3.3$. The time $t$ is scaled with the period $T$ of oscillation.

which, in view of the neglect of flexibility in obtaining this number, is in reasonable agreement with the value obtained from the dynamic experiments.

In order to compare the experimental results with theory, the rotational diffusion at infinite dilution $D_{\mathrm{r}}$ needs to be determined. To do so we determine the Deborah number $\Omega_{\text {eff }}$ for which the limiting values for the phase shifts for $\mathrm{Pe}_{\text {eff }} \rightarrow 0$ as found in the experiments is reproduced. As can be seen in figure 8 there is a reasonable comparison, especially for $\epsilon_{2}\left(P e_{\text {eff }} \rightarrow 0\right)$, between the theoretical calculation using $\Omega_{\text {eff }}=24$ and the experimental frequency of $0.05 \mathrm{~Hz}$ at a concentration of $2 \%$. Since we know from the scaling that $C=$ 3 , it follows that $\frac{L}{d} \varphi=10 / 3$ for this concentration, and thus, with equation (12), we find that $D_{\mathrm{r}}=0.04 \mathrm{~s}^{-1}$. This number, together with the dimensionless concentration $\frac{L}{d} \varphi$, was used in the scaling of the frequency and shear rates in figure 9. In this figure, as well as in figure 8, the theoretical validity of the scaling argument is tested by calculating the dynamic response at two different dimensionless concentrations $\frac{L}{d} \varphi=10 / 3$ and $5 / 3$, having the same distance to the spinodal point as the $2 \%$ and $1 \%$ samples.

The theoretical frequency dependence of phase shifts and nonlinear response functions exhibit the same features as the experimental results, as can be seen from figure 9: the functional form of both is reproduced and the absolute values are in qualitative agreement. Due to the neglect of flexibility, a quantitative agreement is not expected. What is more important, however, is that the functional variation with the effective frequency is the same for both experiment and theory. We can therefore draw the important conclusion that 

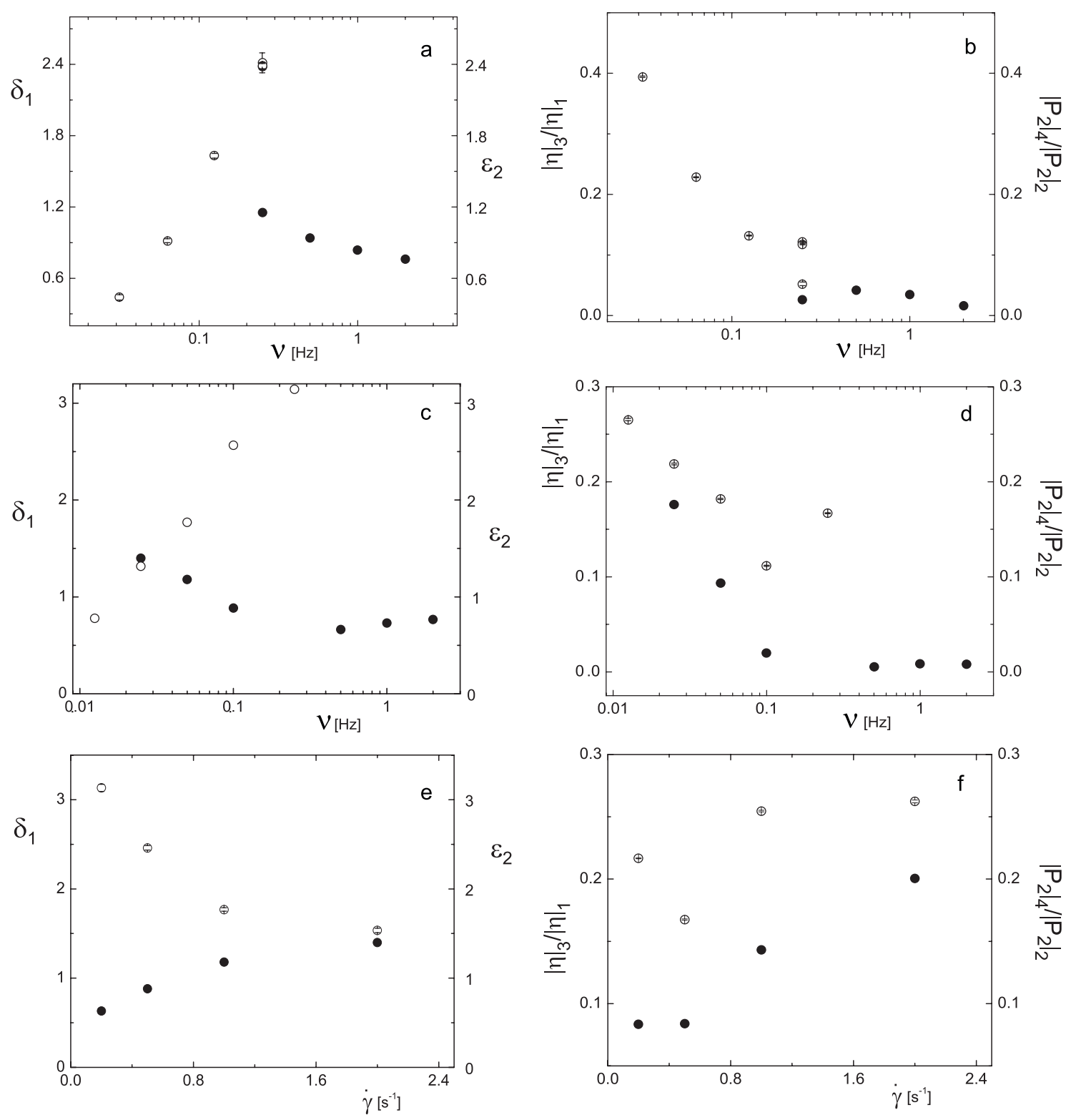

Figure 7. Results of the Fourier analysis using equation (10) for $\left\langle P_{2}\right\rangle(t)$ (open symbols) and equation (9) for the stress (filled symbols) at $1 \%$ ((a) and (b)) and 2\% ((c)-(f)) Pb-Peo. ((a), (c) and (e)) are plots of the phase shift $\delta_{1}$ for the stress and $\epsilon_{2}$ for $\left\langle P_{2}\right\rangle$, while ((b), (d) and (f)) are plots of $|\eta|_{3} /|\eta|_{1}$ for the stress and $\left|P_{2}\right|_{4} /\left|P_{2}\right|_{2}$ for $\left.\left\langle P_{2}\right\rangle\right)$, which quantities measure the departure from linear response. In ((a)-(d)), the shear rate is fixed to $\dot{\gamma}_{\max }=4.0 \mathrm{~s}^{-1}$ for $1 \% \mathrm{~Pb}-\mathrm{Peo}$ and $\dot{\gamma}_{\max }=1.0 \mathrm{~s}^{-1}$ for $2 \% \mathrm{~Pb}-\mathrm{Peo}$. In ((e) and (f)) the frequency is fixed to $v=0.05 \mathrm{~Hz}$.

the scaling with equation (15) in the experiments is justified. In other words, the flow response of the $\mathrm{Pb}-\mathrm{Peo}$ system scales with the distance from the spinodal point.

The correspondence between theory and experiment is especially satisfactory for the frequency dependence of the phase shift in $\left\langle P_{2}\right\rangle, \epsilon_{2}$ in figure 9(c) and the nonlinearity in the stress, given by $|\eta|_{3} /|\eta|_{1}$ in figure 9(b). This correspondence confirms the choice of $D_{\mathrm{r}}=0.04 \mathrm{~s}^{-1}$. The experimental phase shift $\delta_{1}$ in the stress, given in figure 9(a), however, shows a more pronounced frequency dependence as predicted by theory. Concerning the phase shifts, it is interesting to note that, at low frequencies, $\left\langle P_{2}\right\rangle$ is in phase with the applied shear field $\dot{\gamma} \propto \frac{\mathrm{d} \gamma}{\mathrm{d} t} \propto \cos (\omega t)$ and $\epsilon_{2}=0$, while at high frequencies $\epsilon_{2} \rightarrow \pi$. For the stress we observe that at low frequencies $\delta_{1}=$ $\pi / 2$, corresponding to fluid-like behaviour, while $\delta_{1}$ decreases with increasing frequency, but never reaches 0 , which value corresponds to solid-like behaviour. The variation of $\epsilon_{2}$ is twice that of $\delta_{1}$ due to the fact that $\left\langle P_{2}\right\rangle(t) \sim \dot{\gamma}^{2}$, as discussed before.

The frequency dependence of the nonlinear response functions show that with increasing frequency the system becomes more linear (at the cost of an increasing phase shift). The linear response regime therefore extends up to larger shear rates when the frequency increases. The reason for this is that, at high frequencies, microstructural order is not able any more to fully respond to the external field.

There is a considerable discrepancy between the value of the orientational diffusion coefficient $D_{\mathrm{r}}$ at infinite dilution that one would calculate for the length and thickness of the worms from well-known expressions for stiff rods (Tirado et al 1980) and the value found in our experiments. It is unclear 


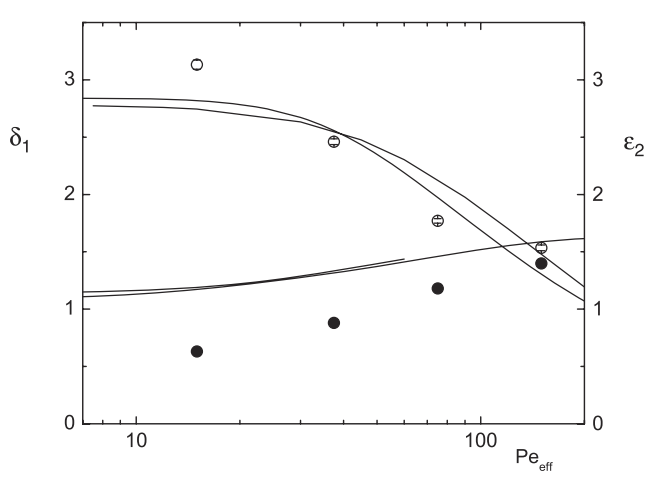

Figure 8. Shear rate dependence of the phase shifts $\delta_{1}$ and $\epsilon_{2}$ for the theoretically calculated response of the stress (bottom curves) and the orientational order parameter $\left\langle P_{2}\right\rangle$ (top curves), respectively, at a scaled volume fraction of $\frac{L}{d} \phi=10 / 3$ (solid line) and $\frac{L}{d} \varphi=5 / 3$ (dashed line). The effective Deborah number is $\Omega=24_{\text {eff. }}$. The symbols give the experimental response for the stress (solid) and $\left\langle P_{2}\right\rangle$ (open) at $2 \% \mathrm{~Pb}-\mathrm{Peo}$, scaled with the orientational diffusion coefficient at infinite dilution with a value of $D_{\mathrm{r}}=0.04 \mathrm{~s}^{-1}$ and $C=3$

whether this is the result of the flexibility of the rods. Another source for this discrepancy might be that the theory neglects dynamical correlations. In the derivation of equations (1) and (7), the rod-rod pair-correlation function is taken equal to the Boltzmann exponential of the pair-interaction potential. This is asymptotically exact for very long and thin hard rods for the calculation of thermodynamic quantities of rod suspensions. For dynamical processes (with or without shear flow), however, such an approximation for the pair correlation is approximate, and particularly neglects dynamical correlations. Simulations have shown that such correlations are of importance, at least for fast dynamical processes (Tao et al 2006). The simulations show that critical slowing down is enhanced by dynamical correlations. This might explain the above-mentioned discrepancy between theory and experiment. This is a subject for future investigations.

\section{Conclusion}

The aim of this paper is to find the microscopic mechanism of the strong shear-thinning behaviour of giant wormlike micelles consisting of $\mathrm{Pb}-\mathrm{Peo}$ block copolymers. The dynamics of the stress is probed by dynamic shear experiments in the linear and nonlinear regimes using Fourier transfer rheology. The dynamics of the orientational order parameter under oscillatory flow is studied with a newly developed timeresolved neutron scattering set-up. It is shown that critical slowing down of orientational Brownian motion due to the vicinity of the isotropic-nematic spinodal is responsible for the shear-thinning behaviour. The response functions for different concentrations are indeed identical when plotted against an effective Deborah number that accounts for critical slowing down. In a certain shear-rate range, shear thinning is so strong that gradient shear banding occurs, where flow profiles have been measured with heterodyne light scattering. The location of the binodal in the shear rate versus concentration plane is determined by step-down rheology, and the spinodal concentration in the absence of flow is obtained from the scaling behaviour of response functions. Both the measured
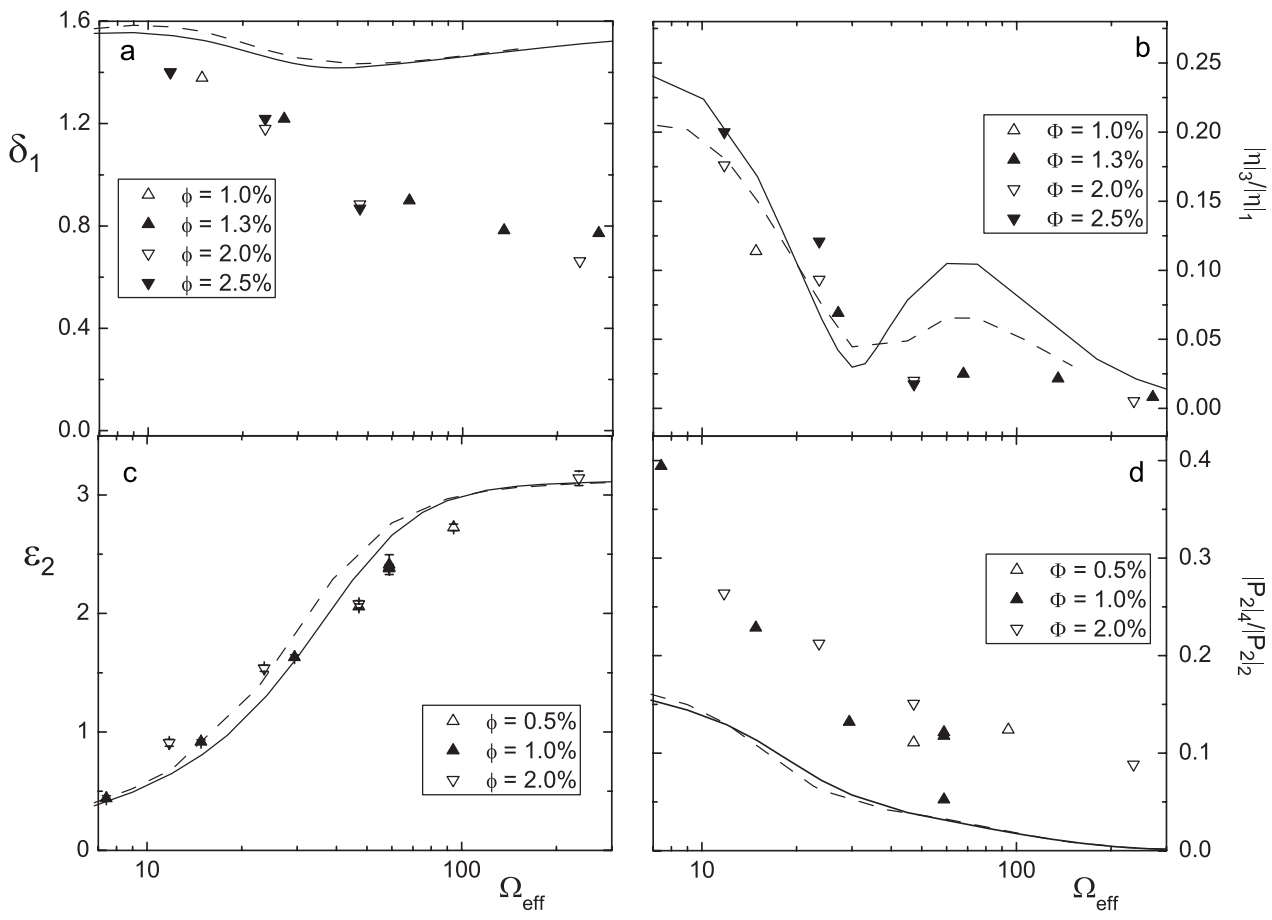

Figure 9. The phase shifts ((a) and (c)) and nonlinearity ((b) and (d)) for the stress ((a) and (b)) and $\left\langle P_{2}\right\rangle((\mathrm{c})$ and (d)) versus the Deborah number. The symbols indicate the experiments for different concentrations. The solid lines give the theoretical responses for $\frac{L}{d} \varphi=10 / 3$ and the dashed line for $\frac{L}{d} \varphi=5 / 3$. For the scaling of the experimental frequency we used a value for the orientational diffusion coefficient at infinite dilution of $D_{\mathrm{r}}=0.04 \mathrm{~s}^{-1}$ and $C=3$ was used, see equation (15). $\mathrm{Pe}_{\text {eff }}=250$ for all data. 
linear and nonlinear stress response and order parameter response are in qualitative agreement with a theory for stiff rods that includes critical slowing down on approach of the isotropic-nematic spinodal. The comparison with theory, however, is qualitative since the theory neglects flexibility. Another possible reason for deviations between theory and experiments might be that the theory neglects dynamical correlations, which have been shown by simulations to enhance critical slowing down. In surfactant wormlike micellar systems, shear thinning can also be due to breaking and stressinduced merging of worms. The breaking and merging of worms can give rise to strong shear thinning by itself, and can give rise to shear banding in the absence of critical slowing down, far away from the spinodal. Scission and stress-induced merging probably do not play a role in the $\mathrm{Pb}-\mathrm{Peo}$ block copolymer system that we studied here.

\section{Acknowledgments}

We acknowledge the Transregio Sonderforschungsbereich TR6018 'Physics of Colloidal Dispersions in External Fields' for financial support. We are grateful to Jörg Stellbrink, Peter Lang and Aggeliki Tsigkri for information on the particle characteristics.

\section{References}

Allgaier J, Poppe A, Willner L and Richter D 1997 Macromolecules 301582
Berret J-F 2004 Molecular Gels (Amsterdam: Elsevier) chapter (Rheophysics of Wormlike Micelles)

Briels W J, Mulder P and den Otter W K 2004 J. Phys.: Condens. Matter 16 S3965-74

Cates M E and Candau S J 1990 J. Phys.: Condens. Matter 26869

Chen Z Y 1993 Macromolecules 263419

Denkova A G, Mendes E and Coppens M-O 2008 J. Phys. Chem. B 112793

Dhont J K G and Briels W J 2003a Colloid Surface A 213131

Dhont J K G and Briels W J 2003b J. Chem. Phys. 1181466

Dhont J K G and Briels W J 2008 Rheol. Acta. 47257

Doi M and Edwards S F 1986 The Theory of Polymer Dynamics (Oxford: Clarendon)

Förster S, Konrad M and Lindner P 2005 Phys. Rev. Lett. 94017803

Keiderling U 2002 Appl. Phys. A 74 S1455

Kohlbrecher J and Wagner W 2000 J. Appl. Cryst. 33804

Lettinga M P and Dhont J K G 2004 J. Phys.: Condens. Matter 16 S 3929

Lund R, Willner L, Richter D and Dormidontova E E 2006 Macromolecules 394566

Manneville S 2008 Rheol. Acta 47 301-18

Olmsted P D 2008 Rheol. Acta. 47283

Picken S J, Aerts J, Visser R and Northolt M G 1990 Macromolecules 233849

Salmon J-B, Colin A and Manneville S 2003 Phys. Rev. Lett. 90228303

Tao Y-G, Dhont J K G and Briels W J 2006 J. Chem. Phys. 124134906

Tirado M, Martinez C and Delatorre J G 1980 J. Chem. Phys. 731986

Wilhelm M, Maring D and Spiess H 1998 Rheol. Acta. 37399

Won Y-Y, Davis H T and Bates F S 1999 Science 283960 